\title{
Scaling of Normal Form Analysis Coefficients Under Coordinate Change
}

\author{
Ian Dobson, Senior Member, IEEE, and Emilio Barocio, Member, IEEE
}

\begin{abstract}
Power system normal form analysis has developed coefficients and indices in modal coordinates to quantify nonlinear modal interactions. We study the changes in the coefficients and indices when the power system equations are expressed in different coordinates or units and show that they can be normalized to be invariant to coordinate changes and thus intrinsic to the power system. The results are illustrated on a 4-generator system. An example shows that the coefficients and indices not only detect nonlinear interactions but also can become very large near a strong resonance in the system linearization.
\end{abstract}

Index Terms-Nonlinear modal behavior, normal form method, power system dynamics, strong resonance.

\section{INTRODUCTION}

$\mathbf{P}$ OWER systems are increasingly operated closer to their limits. Advances in communications, control, computing, signal processing, and power electronics are enabling a more highly controlled power system. Stressed and more highly controlled power systems generally exhibit more nonlinear effects and dynamic modal interactions. To achieve a high performance power system that is controlled to reliably operate near its limits, dynamic modal interactions must be better understood so that they can be mitigated.

Normal form theory is a standard mathematical tool to remove leading order nonlinearities from differential equations by successive nonlinear coordinate changes near an equilibrium [1], [2], [6], [13]. Over the last decade, normal form analysis has been applied to investigate and quantify nonlinear interactions between power system modes [8], [11], [12], [9], [14]. Applications include control system design [8], [7], [3] and predicting interarea separation [11], [12].

Before embarking on a more detailed review of the normal form analysis method in Section II, we make some brief overall introductory remarks. The method starts with power system differential equations which are expanded in a Taylor series about a stable equilibrium. The differential equations are then transformed so that the linearization is diagonalized and the equations are written in modal coordinates. The coefficients of the quadratic terms in the modal coordinates are the quantities $C_{j k}^{i}$, where $i, j$, and $k$ are indices ranging from 1 to $n$, the number

Manuscript received February 27, 2003. This work was supported in part by CONACYT, Mexico, Project 31840-A, by the National Science Foundation (NSF) under Grant ECS-9988574, and by the Power Systems Engineering Research Center (PSerc), an NSF Industry/University Cooperative Research Center.

The authors are with the Electrical and Computer Engineering Department, University of Wisconsin, Madison, WI 53706 USA (e-mail: dobson@engr.wisc.edu; ebarocio@gama.fime.uanl.mx).

Digital Object Identifier 10.1109/TPWRS.2004.831691 of state variables. The $C_{j k}^{i}$ coefficients determine the size of the contributions of mode $j$ and mode $k$ to the differential equation for mode $i$ (see (4) and ([8, Sec. 3.2]). Then the equations are nonlinearly transformed to be linear up to second order. The sizes of second order terms $h 2_{j k}^{i}$ in the nonlinear transformation are used to quantify nonlinear interactions between the modes ([8, Eq. (9)] $\left.)^{1}\right)$. The $h 2_{j k}^{i}$ are calculated from the $C_{j k}^{i}$ using

$$
h 2_{j k}^{i}=\frac{C_{j k}^{i}}{\lambda_{j}+\lambda_{k}-\lambda_{i}}
$$

where $\lambda_{1}, \lambda_{2}, \ldots, \lambda_{n}$ are the eigenvalues of the power system linearization.

A second order resonance occurs when two eigenvalues sum to equal a third eigenvalue. Formula (1) shows that the $h 2_{j k}^{i}$ become large near a second-order resonance in which $\lambda_{j}+\lambda_{k}-\lambda_{i}$ vanishes. Nonlinear mode couplings become large near second order resonance.

When computing a scalar index that describe properties of a power system, one assumes power system equations expressed in certain units or coordinates. It is desirable that the index be invariant (i.e., it does not change) if it is calculated when the same power system is expressed in different units or coordinates. A scalar index that varies depending on the coordinates depends on both the power system and the arbitrary coordinate system used to express the power system equations. In contrast, invariance to coordinate change ensures that the index measures a property of the power system alone; that is, an invariant index is intrinsic to the power system. A good example is the frequency and damping of a particular power system mode of oscillation, these depend only on the power system and do not depend on the coordinates chosen.

When computing a vector index that describes properties of a power system, the index is intrinsic to the power system if it transforms in a standard way when the coordinates are changed. A good example of intrinsic vector indices are eigenvectors of the power system linearization. Similar remarks apply to matrix or tensor indices; the only difference is that the standard coordinate change transformations for matrices and tensors are more elaborate.

In this paper, the effects of coordinate changes and units on normal form coefficients and indices are examined. The coefficients do not transform in a standard way and some of the indices are not invariant to coordinate changes. However, we propose normalizations of the coefficients and indices to fix this

\footnotetext{
${ }^{1}$ One detail to note when reading [8, Eq. (9)] is that (9) is the exact solution of the differential equations (7) that are valid up to second order, but that (9) is not the exact solution of the differential equations (3) that are also valid up to the second order
} 
problem. The normalized coefficients and indices are intrinsic to the power system.

\section{NORMAL Form ANALYsis PROCEDURE}

This section reviews the mechanics of the normal form calculation of Lin, Vittal, Kliemann, and Fouad [8] and Starrett and Fouad [11]. We follow much of the notation of [8] for convenience.

The power system dynamics are linearized about a stable equilibrium and expanded in a Taylor series to obtain

$$
\dot{x}_{i}=\sum_{j=1}^{n} A_{i j} x_{j}+\frac{1}{2} \sum_{j=1}^{n} \sum_{k=1}^{n} H_{j k}^{i} x_{j} x_{k}+\text { h.o.t. }
$$

where $X=\left(x_{1}, x_{2}, \ldots, x_{n}\right)^{t}$ is the system state defined relative to the stable equilibrium, $A$ is the Jacobian matrix and the Hessian $H$ defines the coefficients of quadratic terms. When transients are considered, the initial condition is written as $X_{0}=$ $\left(x_{10}, x_{20}, \ldots, x_{n 0}\right)^{t}$.

The Jacobian matrix $A$ is assumed to be diagonalizable. Equation (2) is transformed to modal coordinates $Y=\left(y_{1}, y_{2}, \ldots, y_{n}\right)^{t}$ which diagonalize the matrix $A$. In particular, $U$ is defined to be a matrix whose columns are the right eigenvectors of $A$. An important detail [8] is that the right eigenvectors (columns of $U$ ) are normalized so that they have length 1. (An assumption about the normalization of $U$ is needed to unambiguously define the $C_{j k}^{i}$ below.) Then

$$
X=U Y
$$

is a transformation to modal $Y$ coordinates. Transforming (2) to the modal $Y$ coordinates yields

$$
\dot{y}_{i}=\lambda_{i} y_{i}+\sum_{j=1}^{n} \sum_{k=1}^{n} C_{j k}^{i} y_{j} y_{k}+\text { h.o.t. }
$$

where $\lambda_{1}, \lambda_{2}, \ldots, \lambda_{n}$ are the eigenvalues of $A$.

The $C_{j k}^{i}$ are the quadratic coefficients in the $Y$ coordinates and are given by

$$
C_{j k}^{i}=\frac{1}{2} \sum_{r=1}^{n} \sum_{\ell=1}^{n} \sum_{m=1}^{n}\left(U^{-1}\right)_{i r} H_{\ell m}^{r} U_{\ell j} U_{m k} .
$$

The $h 2$ coefficients are then defined by (1).

Normal form theory [1], [2], [6], [13] states that the local nonlinear transformation from $Y$ to $Z$ variables given by

$$
y_{i}=z_{i}+\sum_{j=1}^{n} \sum_{k=1}^{n} h 2_{j k}^{i} z_{j} z_{k}
$$

linearizes the differential equations to second order so that

$$
\dot{z}_{i}=\lambda_{i} z_{i}+\text { third order terms. }
$$

An initial condition $X_{0}$ for a transient becomes $Y_{0}=U^{-1} X_{0}$ in the $Y$ coordinates and $Z_{0}$ in the $Z$ coordinates, where $Z_{0}=$ $\left(z_{10}, z_{20}, \ldots, z_{n 0}\right)^{t}$ satisfies

$$
y_{i 0}=z_{i 0}+\sum_{j=1}^{n} \sum_{k=1}^{n} h 2_{j k}^{i} z_{j 0} z_{k 0} .
$$

Several measures of the nonlinearity in $Y$ coordinates were proposed in [8] and [12] based on the $h 2$ coefficients and the initial condition of a transient. An interaction coefficient is defined as

$$
h 2_{j k}^{i} z_{j 0} z_{k 0}
$$

to quantify the effect of second-order terms on the transient solution ([8, Sec. 3.3.2, 3.3.3]). The nonlinear interaction index $I 1$ for mode $i$ is defined as

$$
I 1(i)=\left|y_{i 0}-z_{i 0}+h 2_{j^{*} k^{*}}^{i} z_{j^{*} 0} z_{k^{*} 0}\right|
$$

where the choice of $j=j^{*}$ and $k=k^{*}$ maximizes the interaction coefficient size $\left|h 2_{j k}^{i} z_{j 0} z_{k 0}\right|$ [12, Sec. 2.4]. The nonlinearity index $I 2$ for mode $i$ is defined as ([12, Tables VII, IX], [7, eq. (11)], [14, eq. (10)])

$$
I 2(i)=\frac{h 2_{j^{*} k^{*}}^{i} z_{j^{*} 0} z_{k^{*} 0}}{z_{i 0}} .
$$

We do not address the generalized participation factor analysis of [11] and [15] in this paper.

\section{DEPENDENCE OF COEFFICIENTS AND INDICES ON COORDINATE SYSTEMS AND UNITS}

The coefficients $C_{j k}^{i}, h 2_{j k}^{i}, h 2_{j k}^{i} z_{j 0} z_{k 0}$, and the index $I 1$ depend on the coordinate system used to express the power system differential equations. In particular, the values of these coefficients and indices vary if the state variables are expressed in different units.

\section{A. Simple Example}

The essence of the problem can be seen in a simple example. Consider the following scalar differential equation in which the state variable $x$ is measured in megawatts

$$
\dot{x}=-x+x^{2} .
$$

If we change the state variable to be $\hat{x}$, where $\hat{x}$ is measured in kilowatts, then

$$
\hat{x}=1000 x
$$

and (12) becomes

$$
\dot{\hat{x}}=-\hat{x}+0.001 \hat{x}^{2} .
$$

For (12), it is apparent that $C_{11}^{1}=1 . C_{11}^{1}$ is intended to quantify the nonlinear interaction of mode 1 (the only mode) of system (12) with itself. But in (14), which represents the same physical system in different units, we have $\hat{C}_{11}^{1}=0.001$. It is clear that the size of the nonlinearity as measured by the $C_{11}^{1}$ coefficient varies according to the units chosen to express the differential equation. ${ }^{2}$ Note that both $x$ and $\hat{x}$ are modal coordinates; there is no unique choice of modal coordinates.

\footnotetext{
${ }^{2}$ One way to resolve the issue in this simple one dimensional case is to recognize that the units of $C_{11}^{1}$ are $1 / \mathrm{MJ}$ and the units of $\hat{C}_{11}^{1}$ are $1 / \mathrm{kJ}$. However, consideration of practical power system models with $X$ vectors containing quantities with different units and modal $Y$ vectors containing quantities that are combinations of these units shows that this resolution does not generalize well.
} 
There are also changes in $h 2_{11}^{1}$ and the interaction coefficient $h 2_{11}^{1} z_{0}^{2}$ when the units are changed. For (12), $h 2_{11}^{1}=-1$, whereas for (14), $\widehat{h 2}_{11}^{1}=-0.001$. For (12), solving (8) and (3) gives $z_{0}=(1 / 2)-\sqrt{(1 / 4)-x_{0}}$ and

$$
h 2_{11}^{1} z_{0}^{2}=x_{0}-\frac{1}{2}+\sqrt{\frac{1}{4}-x_{0}}
$$

whereas for $(14), \hat{z}_{0}=500-\sqrt{250000-1000 \hat{x}_{0}}$ and

$$
\begin{aligned}
\widehat{h 2}_{11}^{1} \hat{z}_{0}^{2} & =\hat{x}_{0}-500+\sqrt{250000-1000 \hat{x}_{0}} \\
& =1000 h 2_{11}^{1} z_{0}^{2} .
\end{aligned}
$$

\section{B. The Effect of a Linear Transformation of Coordinates}

We transform the power system differential equations from the original $X$ coordinates to $\hat{X}$ coordinates and then compute the coefficients and indices $\hat{C}_{j k}^{i}, \widehat{h 2}_{j k}^{i}, \widehat{h 2}_{j k}^{i} \hat{z}_{j 0} \hat{z}_{k 0}, \widehat{I 1}, \widehat{I 2}$. In this way, we find out how these coefficients and indices depend on the coordinate system.

Suppose that the $X$ and $\hat{X}$ coordinates are related by an invertible linear transformation $T$ according to

$$
X=T \hat{X} \text {. }
$$

Then the power system equations in $\hat{X}$ coordinates are

$$
\dot{\hat{x}}_{i}=\sum_{j=1}^{n} \hat{A}_{i j} \hat{x}_{j}+\frac{1}{2} \sum_{j=1}^{n} \sum_{k=1}^{n} \hat{H}_{j k}^{i} \hat{x}_{j} \hat{x}_{k}+\text { h.o.t. }
$$

where

$$
\begin{aligned}
\hat{A} & =T^{-1} A T \\
\hat{H}_{j k}^{i} & =\sum_{r=1}^{n} \sum_{\ell=1}^{n} \sum_{m=1}^{n}\left(T^{-1}\right)_{i r} H_{\ell m}^{r} T_{\ell j} T_{m k} .
\end{aligned}
$$

Right eigenvectors in the $\hat{X}$ coordinates are given by the columns of $T^{-1} U$, but in general these columns will not be of length 1 . Therefore, in accordance with the normalization used in Section II, we define

$$
\hat{U}=T^{-1} U N
$$

where $N$ is a diagonal matrix chosen so that $\hat{U}=T^{-1} U N$ is normalized to have columns of length 1 . A formula for the diagonal elements of $N$ is

$$
N_{i i}=\left[\left(U^{t}\left(T^{-1}\right)^{t} T^{-1} U\right)_{i i}\right]^{-\frac{1}{2}} .
$$

An arbitrary choice about the phase and sign of elements of $N$ can be made if it is agreed that only the magnitude of coefficients is of interest. ${ }^{3}$ Now

$$
\hat{C}_{j k}^{i}=\frac{1}{2} \sum_{r=1}^{n} \sum_{\ell=1}^{n} \sum_{m=1}^{n}\left(\hat{U}^{-1}\right)_{i r} \hat{H}_{\ell m}^{r} \hat{U}_{\ell j} \hat{U}_{m k} .
$$

\footnotetext{
${ }^{3}$ Phase or sign of the $h 2$ coefficients is neglected here because no applications or interpretations for this phase have been suggested so far. However, phase could be treated as follows: Additionally normalize the eigenvectors $U$ to have standard phase by, for example, requiring the first component of each eigenvector to have phase zero. Adjust the phase of each $N_{i i}$ so that $\hat{U}$ also has standard phase. Then, following analysis along the lines of this paper, the normalized $h 2$ coefficients (32) would be invariant and phase differences between these normalized $h 2$ coefficients could be meaningful.
}

Substituting from (19) and (20), using (5) and simplifying yields

$$
\hat{C}_{j k}^{i}=\sum_{r=1}^{n} \sum_{\ell=1}^{n} \sum_{m=1}^{n}\left(N^{-1}\right)_{i r} C_{\ell m}^{r} N_{\ell j} N_{m k}
$$

and, since $N$ is diagonal, (22) becomes

$$
\hat{C}_{j k}^{i}=N_{i i}^{-1} C_{j k}^{i} N_{j j} N_{k k} .
$$

Since the eigenvalues are coordinate independent, formula (1) shows that the $h 2_{j k}^{i}$ transform in the same way as the $C_{j k}^{i}$

$$
\widehat{h 2}_{j k}^{i}=N_{i i}^{-1} h 2_{j k}^{i} N_{j j} N_{k k} .
$$

Equations (3), (20), (17) and $\hat{X}=\hat{U} \hat{Y}$ imply that

$$
\hat{Y}=N^{-1} Y \text {. }
$$

If $Y$ and $Z$ satisfy (6), then it can be shown using (24) and (25) that $\hat{Y}$ and $N^{-1} Z$ satisfy

$$
\hat{y}_{i}=\left(N^{-1} z\right)_{i}+\sum_{j=1}^{n} \sum_{k=1}^{n} \widehat{h 2}_{j k}^{i}\left(N^{-1} z\right)_{j}\left(N^{-1} z\right)_{k} .
$$

Therefore

$$
\hat{Z}=N^{-1} Z \text {. }
$$

Equations (27) and (24) imply that the interaction coefficient transforms as

$$
\widehat{h 2}_{j k}^{i} \hat{z}_{j 0} \hat{z}_{k 0}=N_{i i}^{-1} h 2_{j k}^{i} z_{j 0} z_{k 0} .
$$

Since $\widehat{h 2}_{j k}^{i} \hat{z}_{j 0} \hat{z}_{k 0}$ and $h 2_{j k}^{i} z_{j 0} z_{k 0}$ differ by a factor $N_{i i}^{-1}$ that only depends on $i$, the $j$ and $k$ that maximize $\widehat{h 2}_{j k}^{i} \hat{z}_{j 0} \hat{z}_{k 0}$ are the same as the $j$ and $k$ that maximize $h 2_{j k}^{i} z_{j 0} z_{k 0} \cdot j^{*}$ and $k^{*}$ are these maximizing values of $j$ and $k$. and therefore $j^{*}$ and $k^{*}$ do not depend on the coordinate system. Now it follows from (25), (27), and (28) that the indices $I 1$ and $I 2$ transform as

$$
\begin{aligned}
& \widehat{I 1}(i)=N_{i i}^{-1} I 1(i) \\
& \widehat{I 2}(i)=I 2(i) .
\end{aligned}
$$

That is, index $I 1$ varies with the coordinate change and index $I 2$ is invariant to the coordinate change. Note that [5] incorrectly suggests that the index $I 2$ is not invariant.

We consider special cases of the transformation $T$ in which the formula (21) for $N$ becomes simpler.

1) Diagonal $T$ can be interpreted as a transformation that changes the units in which the system states are measured. If $T$ is diagonal, then

$$
N_{i i}=\left(\sum_{j=1}^{n} U_{i j}^{t} U_{j i} T_{j j}^{-2}\right)^{-\frac{1}{2}}
$$

Formula (31) generally simplifies to $N=T$ only when $T$ is a multiple of the identity matrix or $n=1$.

2) If $T$ is orthonormal so that $T^{t} T=I$, then $N=I$ and all the coefficients and indices are invariant under $T$. With the exception of permutations of the system states, orthogonal transformations are of limited use for power systems. 


\section{Discussion}

The transformation formulas (23), (24), and (28) show how the coefficients $C_{j k}^{i}, h 2_{j k}^{i}$, and $h 2_{j k}^{i} z_{j 0} z_{k 0}$ change when the coordinates assumed for the power system equations are changed by a linear transformation. Since the transformation formulas depend on the matrix $N$ which normalizes the columns of $U$, these coefficients are neither independent of the coordinates assumed for the differential equations, nor do they transform as a tensor with respect to those coordinates. Thus, the coefficients $C_{j k}^{i}, h 2_{j k}^{i}$, and $h 2_{j k}^{i} z_{j 0} z_{k 0}$ are not intrinsic to the power system. This can be problematic for quantifying nonlinear interactions with these coefficients. For example, the ranking of these coefficients by size can change when different coordinates are used. Another problem is that when the operating point changes, the scaling of the coefficients changes (because the eigenvectors $U$ change and this changes $N$ via (21)). This arbitrary change in scaling muddles comparisons of coefficients obtained at different operating points of the same power system.

For a general linear transformation of the coordinates assumed for the power system equations, the coefficients $C_{j k}^{i}, h 2_{j k}^{i}$, and $h 2_{j k}^{i} z_{j 0} z_{k 0}$ transform in way that depends in a complicated fashion on the linear transformation. A similarly complicated dependence would also arise in the case of a nonlinear coordinate transformation such as changing phasors from polar coordinates to rectangular coordinates.

A diagonal transformation of coordinates $T$ (case 1 in Section III-B) is an important special case because it represents an independent rescaling of each of the state variables, or, equivalently, a change in units. In this special case, the $N$ matrix is given by (31) and the coefficients have the nontrivial transformations (23), (24), and (28). In power system models, there is a mixed set of units because the state variables include different physical quantities and these transformations show how the coefficients change when, for example, angles are measured in degrees instead of radians, or when per unit scaling is introduced or removed for some of the states.

One perspective to help explain the transformations of the coefficients $C_{j k}^{i}, h 2_{j k}^{i}$, and $h 2_{j k}^{i} z_{j 0} z_{k 0}$ is as follows: The idea of these coefficients is to transform the system to a standard coordinate system, namely modal coordinates that diagonalize the Jacobian, and to measure the nonlinear interactions in that particular coordinate system. However, there are no unique modal coordinates; any given set of modal coordinates can be independently rescaled to yield another set of modal coordinates. (There appears to be no intrinsic way to select one of these sets of modal coordinates as canonical.) The scaling of the modal coordinates is chosen by the scaling of the eigenvectors (columns of $U$ ). Different coordinate systems for the original power system equations yield different choices of scalings for the modal coordinates [see (25)] and hence different values of the coefficients.

The $I 2$ index is a constant independent of the coordinate system [see (30)], but the $I 1$ index varies with the coordinate system [see (29)]. Recent work applying normal form analysis [15] mentions the difficulty of quantifying nonlinearity with the $I 1$ and $I 2$ indices in comparing cases at different operating conditions: "However, using these indices, it was difficult to compare the nonlinearity quantitatively because of the scaling of the eigenvectors." This statement is consistent with our results for the $I 1$ index.

\section{Normalized Coefficients and Indices}

The lack of invariance of $h 2_{j k}^{i}, h 2_{j k}^{i} z_{j 0} z_{k 0}$, and $I 1$ described above can be fixed by redefining these coefficients and indices with suitable normalization. A normalized interaction coefficient is

$$
\overline{h 2}_{j k}^{i} z_{j 0} z_{k 0}=\frac{h 2_{j k}^{i} z_{j 0} z_{k 0}}{z_{i 0}} .
$$

Normalization (32) is consistent with the way that Thapar et al. [12] advise that the magnitude of $h 2_{j k}^{i} z_{j 0} z_{k 0}$ should be assessed relative to the magnitude of $z_{i 0}$.

A normalized index $I 1$ is

$$
\begin{aligned}
\overline{I 1}(i) & =\frac{\left|y_{i 0}-z_{i 0}+h 2_{j^{*} k^{*}}^{i} z_{j^{*} 0} z_{k^{*} 0}\right|}{\left|z_{i 0}\right|} \\
& =\left|\frac{y_{i 0}}{z_{i 0}}-1+\overline{h 2} i_{j^{*} k^{*}} z_{j^{*} 0} z_{k^{*} 0}\right| .
\end{aligned}
$$

The new coefficient (32) and index (33) are constants invariant under linear coordinate change and are intrinsic to the power system. ${ }^{4}$ The normalized index $\overline{I 1}(i)$ in (33) can be interpreted as the index $I 1(i)$ measured in multiples of the size of the initial disturbance in mode $i$.

\section{Numerical Results on 4-GENERATOR System}

This section computes unnormalized and normalized coefficients and indices on the 4-generator power system shown in Fig. 1. The system was obtained from [10] and a static var compensator was added at bus 8 to improve the voltage profile. The generators are represented by a two-axis model with simple excitation control. Generator, svc, and line data are described in Appendix A. There are two identical areas, each with two 900 MVA generators. Generators 1, 2, 4 are loaded to 700 MVA and slack generator 3 is loaded to 751 MVA. Area 1 and area 2 are loaded to 967 and $1767 \mathrm{MW}$, respectively. This base case is a stressed condition.

Selected modes of interest are shown in Table I. Mode 1 is associated with the static var compensator. Modes 14, 15, 19 are control modes, mode 16 is associated with flux in generators 3 and 4, and modes 6-11 are electromechanical modes.

A transient was produced by a solid fault applied at bus 10 and cleared in $0.2 \mathrm{~s}$. The normal form analysis described in Section II was used to compute the unnormalized interaction coefficients for this transient. Table II shows the magnitude of selected interaction coefficients for modes of interest. The unnormalized interaction coefficients in column 4 of Table II were computed with angle variables expressed in radian. Changing the units of angles from radian to degrees is a simple example of a coordinate change. For this coordinate change, the transformation matrix $T$ is diagonal with entries that are $(180 / \pi)$ when the corresponding state variable is an angle and 1 otherwise. Formulas (21) and (24) were used to compute the effects of this coordinate change and the resulting unnormalized normal-

\footnotetext{
${ }^{4}$ An alternative normalization of (32) and (33) dividing by $\left|y_{i 0}\right|$ instead of $\left|z_{i 0}\right|$ also yields invariance under linear coordinate change.
} 


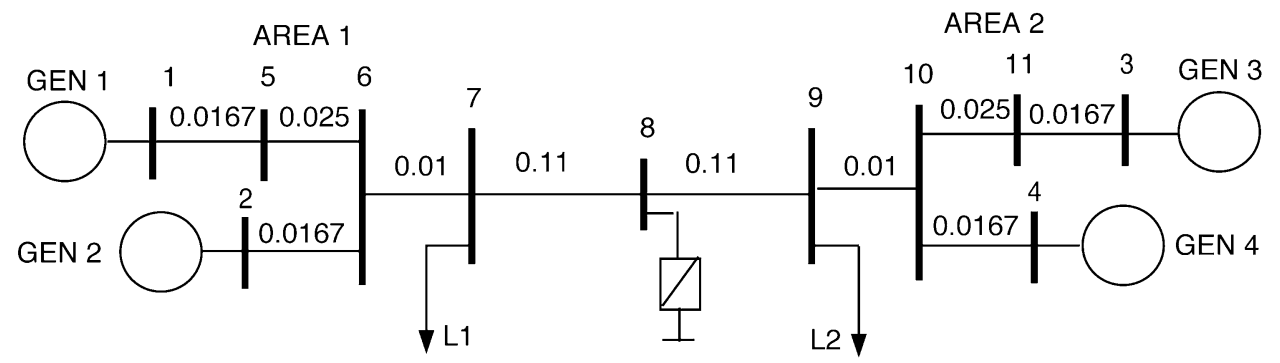

Fig. 1. Two-area, 4-generator system (reactances in pu with 100 MVA base).

TABLE I

EIGENVALUES OF 4-GENERATOR SYSTEM

\begin{tabular}{ccl}
\hline mode & eigenvalue $(\mathrm{rad} / \mathrm{s})$ & \\
\hline 1 & -101.17 & svc \\
6,7 & $-0.801 \pm 6.895 \mathrm{j}$ & local mode area 1 \\
8,9 & $-0.762 \pm 6.889 \mathrm{j}$ & local mode area 2 \\
10,11 & $-0.030 \pm 2.628 \mathrm{j}$ & interarea \\
14,15 & $-9.385 \pm 0.873 \mathrm{j}$ & control \\
16 & -6.598 & flux mode area 2 \\
19 & -3.983 & control \\
\hline
\end{tabular}

TABLE II

INTERACTION COEFFICIENTS $\left|h 2_{j k}^{i} z_{j o} z_{k o}\right|$

\begin{tabular}{cccccc}
\hline & $j$ & $k$ & angles & angles & \\
in rad & in deg & normalized \\
\hline 1 & 1 & 10 & 3.008 & 3.008 & 2.258 \\
6,7 & 16 & 19 & 0.018 & 0.012 & 0.261 \\
8,9 & 16 & 19 & 0.075 & 0.061 & 0.209 \\
10,11 & 10 & 11 & 0.147 & 0.103 & 0.091 \\
14,15 & 16 & 16 & 42.42 & 40.18 & 2.870 \\
16 & 16 & 16 & 10.54 & 10.54 & 0.338 \\
19 & 16 & 19 & 0.458 & 0.458 & 0.146 \\
\hline
\end{tabular}

TABLE III

INDEX $I 1$

\begin{tabular}{cccc}
\hline mode & $\begin{array}{c}\text { angles } \\
\text { in rad }\end{array}$ & $\begin{array}{c}\text { angles } \\
\text { in deg }\end{array}$ & normalized \\
\hline 1 & 5.182 & 5.182 & 3.891 \\
6,7 & 0.023 & 0.035 & 0.499 \\
8,9 & 0.099 & 0.121 & 0.336 \\
10,11 & 0.211 & 0.302 & 0.187 \\
14,15 & 56.70 & 59.85 & 4.050 \\
16 & 15.138 & 15.139 & 0.485 \\
19 & 0.962 & 0.962 & 0.307 \\
\hline
\end{tabular}

ized interaction coefficients with angles in degrees are shown in column 5 of Table II. The coordinate change makes a noticeable change to the interaction coefficients. The normalized interaction coefficients were computed using (32) and are shown in the last column of Table II. There is a significant difference in the ranking of the interaction coefficient magnitudes when comparing the unnormalized and normalized coefficients.

The index $I 1$ was computed for the same transient and the same modes and the results are shown in Table III. The second column of Table III shows the unnormalized index $I 1$ with angle variables expressed in radian and the third column of Table III shows the unnormalized index $I 1$ with angle variables expressed in degrees. The coordinate change makes a noticeable change to the index, with the largest change occurring in mode 14, 15 and the largest percentage change occurring in mode 10, 11. The last column of Table III shows the normalized index $I 1$ for the same modes. Normalization of
$I 1$ indices for the selected modes significantly changes their ranking and relative magnitudes.

\section{NORMAL Form ANALYSIS NEAR StRONG RESONANCE}

If two eigenvalues of the power system linearization coincide and the linearization is not diagonalizable, this is called strong resonance. Dobson et al. [4] explains strong resonance, reviews the literature, and gives an example of proximity to strong resonance leading to oscillations in a power system example. In the context of normal form analysis, strong resonance is a first order resonance occurring within the power system linearization.

Consider the following example:

$$
\left(\begin{array}{l}
\dot{x}_{1} \\
\dot{x}_{2} \\
\dot{x}_{3} \\
\dot{x}_{4}
\end{array}\right)=\left(\begin{array}{cccc}
-1 & 1 & 1 & 0 \\
\mu & -1 & 0 & 1 \\
-1 & 0 & -1 & 1 \\
0 & -1 & \mu & -1
\end{array}\right)\left(\begin{array}{l}
x_{1} \\
x_{2} \\
x_{3} \\
x_{4}
\end{array}\right)+\left(\begin{array}{c}
0 \\
\frac{1}{2} \epsilon x_{1}^{2} \\
0 \\
0
\end{array}\right) .
$$

All $H_{j k}^{i}=0$ except that $H_{11}^{2}=\epsilon . \epsilon$ and $\mu$ are small real constants. The eigenvalues of the matrix in (34) are $\lambda_{1}=-1+i+$ $\sqrt{\mu}, \lambda_{2}=-1+i-\sqrt{\mu}$ and their complex conjugates $\lambda_{1}^{*}, \lambda_{2}^{*}$. The eigenvalues $\lambda_{1}$ and $\lambda_{2}$ coincide in a strong resonance at $-1+i$ when $\mu=0$. We assume $\mu \neq 0$ in order to diagonalize the matrix in (34) and compute

$$
\begin{aligned}
U & =\frac{1}{\sqrt{2} \sqrt{1+\mu}}\left(\begin{array}{cccc}
1 & 1 & 1 & 1 \\
\sqrt{\mu} & -\sqrt{\mu} & \sqrt{\mu} & -\sqrt{\mu} \\
i & i & -i & -i \\
i \sqrt{\mu} & -i \sqrt{\mu} & -i \sqrt{\mu} & i \sqrt{\mu}
\end{array}\right) \\
U^{-1} & =\frac{\sqrt{1+\mu}}{2 \sqrt{2}}\left(\begin{array}{cccc}
1 & \frac{1}{\sqrt{\mu}} & -i & \frac{-i}{\sqrt{\mu}} \\
1 & \frac{-1}{\sqrt{\mu}} & -i & \frac{i}{\sqrt{\mu}} \\
1 & \frac{1}{\sqrt{\mu}} & i & \frac{i}{\sqrt{\mu}} \\
1 & \frac{-1}{\sqrt{\mu}} & i & \frac{-i}{\sqrt{\mu}}
\end{array}\right) .
\end{aligned}
$$

Then (34) in $Y$ coordinates becomes

$$
\begin{aligned}
\left(\begin{array}{l}
\dot{y}_{1} \\
\dot{y}_{2} \\
\dot{y}_{3} \\
\dot{y}_{4}
\end{array}\right)= & \left(\begin{array}{l}
\lambda_{1} y_{1} \\
\lambda_{2} y_{2} \\
\lambda_{1}^{*} y_{3} \\
\lambda_{2}^{*} y_{4}
\end{array}\right) \\
& +\frac{\epsilon\left(y_{1}+y_{2}+y_{3}+y_{4}\right)^{2}}{8 \sqrt{2} \sqrt{\mu} \sqrt{1+\mu}}\left(\begin{array}{c}
1 \\
-1 \\
1 \\
-1
\end{array}\right)
\end{aligned}
$$

and

$$
h 2_{j k}^{i}=\frac{\epsilon(-1)^{i+1}}{8\left(\lambda_{j}+\lambda_{k}-\lambda_{i}\right) \sqrt{2} \sqrt{\mu} \sqrt{1+\mu}} .
$$


Near the strong resonance, $h_{j k}^{i}$ scales as $(\epsilon / \sqrt{\mu})$. Here, $\epsilon$ controls the amount of nonlinearity in the original coordinates $(\epsilon=$ 0 gives no nonlinearity) and $\mu$ controls the proximity to strong resonance ( $\mu=0$ gives a strong resonance). No matter how small $\epsilon$ is, one can choose a smaller $\mu$ so that the $h_{j k}^{i}$ have very large magnitudes. Indeed $\left|h_{j k}^{i}\right|$ tends to infinity as the strong resonance is approached. Appendix B proves that as $\mu$ tends to zero, one or more of the interaction coefficients $h 2_{j k}^{i} z_{j 0} z_{k 0}$ becomes arbitrarily large. Indices $I 1$ and $I 2$ become arbitrarily large in the same way.

That is, no matter how small is the quadratic nonlinearity in the equations in the original $X$ coordinates, one can, by moving close to the strong resonance, make the coefficients and indices $h_{j k}^{i}, h 2_{j k}^{i} z_{j 0} z_{k 0}, I 1, I 2$ arbitrarily large. Therefore caution is needed in interpreting these coefficients and indices near strong resonance. Large values of these coefficients and indices correctly reflect the high nonlinearity of the system in the modal $Y$ coordinates, but do not necessarily imply that the system has significant nonlinearity in the original $X$ coordinates. The coordinate change to modal $Y$ coordinates requires increasing distortion as strong resonance is approached and this distortion can greatly amplify nonlinearities.

\section{CONCLUSION}

We examine how the normal form analysis coefficients $C_{j k}^{i}, h_{j k}^{i}$, and $h 2_{j k}^{i} z_{j 0} z_{k 0}$ vary when the power system differential equations are expressed in different coordinates or different units. Under a general linear coordinate change, these coefficients are not constants and do not transform as tensors. Thus the coefficients are not intrinsic to the system. This can be problematic for quantifying nonlinear interactions with these coefficients. The behavior of the coefficients under coordinate change is related to the nonuniqueness of modal coordinates and the eigenvector scaling induced by the coordinates. The dependence of the index $I 1$ on the coordinate system can cause $I 1$ to vary in a nonstandard way. We correct an error in [5] by showing that the index $I 2$ is invariant to coordinate changes. (That is, $I 2$ as previously defined [12] is already normalized.) Numerical results on a 4 bus generator system show that a coordinate change such as changing the units of angles from radian to degree can produce a noticeable change in unnormalized coefficients and indices.

We suggest a normalized interaction coefficient (32) and a normalized $I 1$ index (33). The effect of the normalization on ranking and magnitudes of these indices and coefficients can be significant. The normalized interaction coefficient and normalized $I 1$ index are invariant under coordinate change and intrinsic to the system. The normalized interaction coefficient and normalized $I 1$ index describe aspects of the power system dynamics more accurately because they do not depend on an arbitrary choice of coordinate system. Therefore, when it is appropriate to apply these indices and coefficients, we recommend that the normalized versions be used.

An example shows that normal form analysis coefficients and indices can become arbitrarily large near strong resonance despite very small amounts of nonlinearity in the system expressed in its original coordinates. Near strong resonance, the transfor-
TABLE IV

LINE IMPEDANCE DATA (PU, BASE 100 MVA)

\begin{tabular}{ccccc}
\hline bus & bus & resistance & reactance & line charging \\
\hline 1 & 5 & 0 & 0.0167 & 0 \\
5 & 6 & 0.0025 & 0.0250 & 0.04375 \\
2 & 6 & 0 & 0.0167 & 0 \\
6 & 7 & 0.001 & 0.01 & 0.0175 \\
7 & 8 & 0.011 & 0.11 & 0.1925 \\
8 & 9 & 0.011 & 0.11 & 0.1925 \\
9 & 10 & 0.001 & 0.01 & 0.0175 \\
4 & 10 & 0 & 0.0167 & 0 \\
10 & 11 & 0.0025 & 0.0250 & 0.04375 \\
3 & 11 & 0 & 0.0167 & 0 \\
(line charging is the total for both ends of the line) \\
\hline \multicolumn{4}{c}{}
\end{tabular}

TABLE V

LOAD FLOW Bus DATA (PU, BASE 100 MVA)

\begin{tabular}{ccccccc}
\hline & & \multicolumn{2}{c}{ Generation } & \multicolumn{2}{c}{ Load } \\
Bus & Voltage & Angle & real & reactive & real & reactive \\
\hline 1 & 1.0300 & 56.1902 & 7.0000 & 2.5852 & 0 & 0 \\
2 & 1.0100 & 46.2257 & 7.0000 & 4.1094 & 0 & 0 \\
3 & 1.0300 & 0 & 7.5055 & 3.0453 & 0 & 0 \\
4 & 1.0100 & -11.3538 & 7.0000 & 4.9033 & 0 & 0 \\
5 & 0.9946 & 49.6377 & 0 & 0 & 0 & 0 \\
6 & 0.9491 & 39.2213 & 0 & 0 & 0 & 0 \\
7 & 0.9091 & 30.2063 & 0 & 0 & 9.67 & 1.0 \\
8 & 0.9536 & 0.1918 & 0 & 2.7536 & 0 & 0 \\
9 & 0.8882 & -28.0995 & 0 & 0 & 17.67 & 1.0 \\
10 & 0.9361 & -18.4562 & 0 & 0 & 0 & 0 \\
11 & 0.9881 & -7.0740 & 0 & 0 & 0 & 0 \\
\hline
\end{tabular}

TABLE VI

LINE FLOWS (PU, BASE 100 MVA)

\begin{tabular}{ccrr}
\hline bus & bus & real & reactive \\
\hline 1 & 5 & 7.000 & 2.5852 \\
5 & 6 & 7.000 & 1.7086 \\
2 & 6 & 7.000 & 4.1094 \\
6 & 7 & 13.8686 & 3.4667 \\
7 & 8 & 3.9717 & 0.2128 \\
8 & 9 & 3.7606 & 1.0228 \\
9 & 10 & -14.0954 & -1.6736 \\
4 & 10 & 7.000 & 4.9033 \\
10 & 11 & -7.3507 & -0.5049 \\
3 & 11 & 7.5055 & 3.0453 \\
\hline
\end{tabular}

mation to modal coordinates introduces a large nonlinearity into the system and this large nonlinearity is quantified by the coefficients. That is, as well as quantifying second-order nonlinear modal interactions, normal form analysis coefficients and indices detect strong resonance effects (first order linear modal interactions) that are not necessarily related to significant system nonlinearity in the original coordinates. Care is warranted in interpreting large values of these coefficients and indices as evidence of system nonlinearity when eigenvalues are close together.

\section{APPENDIX A \\ SYSTEM DATA}

Each machine has $X_{d}=1.8, X_{q}=1.7, T_{d o}^{\prime}=8.0, T_{q o}^{\prime}=$ $0.4, x_{d}^{\prime}=x_{q}^{\prime}=0.3, H=6.5, D=3.0$. Each exciter has $K_{\text {exc }}=100, T_{\text {exc }}=0.02$. The machine data are in per unit (base 900 MVA) except that time constants are in second. The static var compensator model [3] has $K_{\mathrm{svc}}=50, T_{\mathrm{svc}}=0.05$. The line data is shown in Table IV. The base case load flow data is shown in Table VI. Bus 3 is the slack bus and the loads are constant power loads. The system frequency is $60 \mathrm{~Hz}$. The line 
and svc data are in per unit (base 100 MVA) except that time constants are in seconds and angles are in degrees.

\section{APPENDIX B}

Assume that $X_{0} \neq 0$ and $\epsilon \neq 0$. We show that at least one of $\left|h 2_{j k}^{i} z_{j 0} z_{k 0}\right| \rightarrow \infty$ as $\mu \rightarrow 0$. Equation (8) can be written as

$$
\left(\begin{array}{l}
Z_{0}^{t} h 2^{1} Z_{0} \\
Z_{0}^{t} h 2^{2} Z_{0} \\
Z_{0}^{t} h 2^{3} Z_{0} \\
Z_{0}^{t} h 2^{4} Z_{0}
\end{array}\right)=U^{-1} X_{0}-Z_{0}
$$

Since the minimum singular value of $U^{-1}$ is $\sqrt{1+\mu} / \sqrt{2},\left|U^{-1} X_{0}\right| \quad \geq \quad(\sqrt{1+\mu} / \sqrt{2})\left|X_{0}\right| \quad$ and $\left|U^{-1} X_{0}\right| \rightarrow \infty$ as $\mu \rightarrow 0$. If $Z_{0}$ is bounded as $\mu \rightarrow 0$, then the left hand side of (37) is unbounded as $\mu \rightarrow 0$, and therefore at least one of $\left|h 2_{j k}^{i} z_{j 0} z_{k 0}\right| \rightarrow \infty$ as $\mu \rightarrow 0$. If $Z_{0}$ is unbounded as $\mu \rightarrow 0$, then there is an $i$ with $\left|z_{i 0}\right| \rightarrow \infty$ as $\mu \rightarrow 0$. Since, according to (36), $\left|h 2_{i i}^{i}\right| \rightarrow \infty$ as $\mu \rightarrow 0,\left|h 2_{i i}^{i} z_{i 0} z_{i 0}\right| \rightarrow \infty$ as $\mu \rightarrow 0$.

\section{ACKNOWLEDGMENT}

The authors thank M. Gibbard and D. Vowles for helpful comments.

\section{REFERENCES}

[1] V. I. Arnold, Geometrical Methods in the Theory of Ordinary Differential Equations. New York: Springer-Verlag, 1988.

[2] D. K. Arrowsmith and C. M. Place, An Introduction to Dynamical Systems. Cambridge, U.K.: Cambridge Univ. Press, 1990.

[3] E. Barocio and A. R. Messina, "Analysis of nonlinear modal interaction in stressed power systems with SVCs," in Proc. IEEE Power Engineering Society Winter Meeting, New York, Jan. 2002.

[4] I. Dobson, J. Zhang, S. Greene, H. Engdahl, and P. W. Sauer, "Is strong modal resonance a precursor to power system oscillations?," IEEE Trans. Circuits Syst. I, pt. 1, vol. 48, pp. 340-349, Mar. 2001.

[5] I. Dobson, "Strong resonance effects in normal form analysis and subsynchronous resonance," in Proc. IREP Conf. Bulk Power System Dynamics and Control V, Onimichi, Japan, Aug. 2001.

[6] J. Guckenheimer and P. Holmes, Nonlinear Oscillations, Dynamical Systems, and Bifurcations of Vector Fields. New York: Springer-Verlag, 1986.

[7] G. Jang, V. Vittal, and W. Kliemann, "Effect of nonlinear modal interaction on control performance: Use of normal forms technique in control design, Part I: General theory and procedure, and Part II: Case studies," IEEE Trans. Power Syst., vol. 13, pp. 401-413, May 1998.
[8] C.-M. Lin, V. Vittal, W. Kliemann, and A. A. Fouad, "Investigation of modal interaction and its effects on control performance in stressed power systems using normal forms of vector fields," IEEE Trans. Power Syst., vol. 11, pp. 781-787, May 1996.

[9] Y.-X. Ni, V. Vittal, W. Kliemann, and A. A. Fouad, "Nonlinear modal interactions in HVDC/AC power systems with DC power modulation," IEEE Trans. Power Syst., vol. 11, pp. 2011-2017, Nov. 1996.

[10] G. Rogers, Power System Oscillations. Boston, MA: Kluwer, 2000.

[11] S. K. Starrett and A. A. Fouad, "Nonlinear measures of mode-machine participation," IEEE Trans. Power Syst., vol. 13, pp. 389-394, May 1998.

[12] J. Thapar, V. Vittal, W. Kliemann, and A. A. Fouad, "Application of the normal form of vector fields to predict interarea separation in power systems," IEEE Trans. Power Syst., vol. 12, pp. 844-850, May 1997.

[13] S. Wiggins, Introduction to Applied Nonlinear Dynamical Systems and Chaos. New York: Springer-Verlag, 1990.

[14] S. Zhu, V. Vittal, and W. Kliemann, "Analyzing dynamic performance of power systems over parameter space using normal forms of vector fields Part I: Identification of vulnerable regions (republished)," IEEE Trans. Power Syst., vol. 16, pp. 711-718, Nov. 2001.

[15] — "Analyzing dynamic performance of power systems over parameter space using normal forms of vector fields Part II: Comparison of system structure," IEEE Trans. Power Syst., vol. 16, pp. 451-455, Aug. 2001

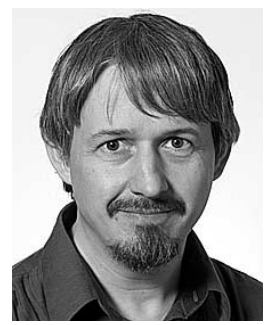

Ian Dobson received the B.A. degree in mathematics from Cambridge, U.K., in 1978 and the Ph.D. degree in electrical engineering from Cornell University in 1989.

He worked from 1978 to 1983 as a Systems Analyst for the British firm EASAMS, Ltd. In 1989, he joined the University of Wisconsin, Madison, where he is now Professor in electrical and computer engineering. His current interests are applications of complex systems and nonlinear dynamics, cascading failure and self organized criticality in blackout risk, electric power system instabilities, and power electronics.

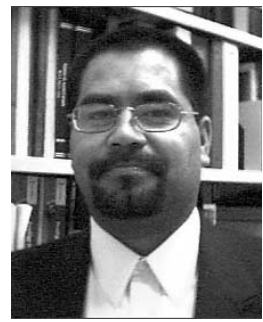

Emilio Barocio received the M.S. degree from the University of Guadalajara, Mexico, in 1997, and the $\mathrm{Ph} . \mathrm{D}$. degree from the Centre for Research and Advanced Studies (CINVESTAV) of the National Polytechnic Institute (IPN) of Mexico in 2003.

From 1997 to 2002, he was a Lecturer in the Department of Mathematics at the University of Guadalajara. He was a Visiting Scholar at the University of Wisconsin,Madison, in the academic year 2001-2002. Currently he is Associate Professor in the Postgraduate Mechanical and Electrical Engineering School of the Autonomous University of Nuevo Léon, Mexico. Dr. Barocio is a member of the Mexican National Research System. 